\title{
Les pratiques enseignantes mises en œuvre en contexte de classe autochtone universitaire : analyse du discours du corps enseignant d'une université québécoise
}

Gabriel Gingras-Lacroix

Université du Québec en Abitibi-Témiscamingue

Oscar Labra

Université du Québec en Abitibi-Témiscamingue

\section{Résumé}

Cette recherche qualitative phénoménologique réalisée auprès de dix membres du corps enseignant de l'Université du Québec en Abitibi-Témiscamingue s'intéresse aux pratiques enseignantes utilisées pour favoriser la réussite des étudiants autochtones. Il résulte de l'analyse thématique du discours des participants, collecté par des entrevues semi-dirigées, que leurs choix pédagogiques ne dépendent pas de la présence ou non d'étudiants autochtones dans le groupe. Cette étude révèle également que, dans ce contexte, les participants adaptent le contenu de leurs cours et adoptent une attitude d'écoute, de

Canadian Journal of Education / Revue canadienne de l'éducation 44:4 (2021)

(C)2021 Canadian Society for the Study of Education/ Société canadienne pour l'étude de l'éducation 
flexibilité, d'ouverture et de respect. Cela témoigne des efforts individuels déployés pour rendre l'enseignement universitaire plus inclusif.

Mots-clés : pédagogie universitaire, pratiques enseignantes, étudiants autochtones, apprentissages holistiques, corps enseignant universitaire

\begin{abstract}
This phenomenological qualitative research conducted among ten members of the teaching staff of the University of Quebec in Abitibi-Témiscamingue studied teaching practices used to support the success of indigenous students. It results from the thematic analysis of the participants' discourse, collected through semi-structured interviews, that their pedagogical choices do not depend on the presence of indigenous students in the group. This study also shows that, in this context, participants adapt the content of their courses and adopt an attitude of listening, flexibility, openness and respect. This reflects individual efforts made to make university education more inclusive of indigenous students.
\end{abstract}

Keywords: university pedagogy, teaching practices, indigenous students, holistic learning, university teaching staff 


\section{Introduction}

Les universités québécoises sont depuis plusieurs années confrontées à de nombreuses mutations les menant à offrir des services pédagogiques novateurs et à adopter des pratiques enseignantes adaptées aux étudiants et aux attentes de la société (Nayyar, 2008). Cette vision néolibérale de l'éducation ayant comme visée première l'employabilité, celle-ci fait référence à un modèle d'éducation eurocentrique (Bousquet, 2008; Giroux, 2010; Nayyar, 2008; Sanford et al., 2012; St-Onge, 2016; Watkins, 2008). Or, si plusieurs mobilisations autochtones ont permis aux Premiers Peuples d'avoir une plus grande autorité en éducation (Commission de l'éducation, 2007; Fraternité des Indiens du Canada, 1972), l'obtention d'un diplôme universitaire oblige toujours les étudiants autochtones à intégrer une structure universitaire occidentale.

\section{Problématique}

Le dernier recensement canadien montrait que le taux de diplomation universitaire de l'ensemble des populations autochtones ${ }^{1}$ est de $11 \%$, alors qu'il se situe à $29 \%$ chez l'ensemble de la population canadienne (Statistique Canada, 2016). Cet écart reposerait notamment sur plusieurs facteurs historiques, socioéconomiques et culturels (Archambault, 2010; Colomb, 2012). L'un de ces facteurs serait lié aux méthodes pédagogiques fréquemment utilisées dans les milieux universitaires (Colomb, 2012; Timmons et al., 2009). Selon Romainville et Michaut (2012), la qualité des pratiques pédagogiques utilisées par le corps enseignant universitaire a un impact non négligeable sur la persévérance scolaire des étudiants. Or, Kermoal (2018) souligne que l'autochtonisation des universités nécessite que ces dernières amorcent une réflexion approfondie concernant les valeurs promues, les rapports de pouvoir et les privilèges qui sont présents au sein de leur établissement.

Plusieurs communautés autochtones partagent une vision semblable de l'éducation (Augustus, 2015), à savoir que celle-ci devrait : 1) accorder une importance particulière aux territoires; 2) impliquer la communauté et la famille dans le processus

1 Membres des Premières Nations, Métis et Inuit (Statistiques Canada, 2016). 
d'apprentissage; 3) promouvoir les langues autochtones; 4) utiliser des approches pédagogiques holistiques; 5) favoriser un processus de décolonisation (Anuik et Gillies, 2012; Battiste, 2008; Battiste et Henderson, 2009; Black et Hachkowski, 2018; Borrows, 2016; Colomb, 2012; Lévesque et al., 2015; Loiselle et Legault, 2010; Munroe et al., 2013; Sioui, 2012; Styres et al., 2013). Comme certaines de ces communautés adoptent la pensée circulaire, basée sur une éthique qui promeut l'existence d'interrelations entre toutes choses existantes et/ou subjectives (Sioui, 2008), une approche holistique ${ }^{2}$ en éducation s'avère indiquée au contexte de classe autochtone (Borrows, 2016; Price et Burtch, 2010).

Selon Battiste (2013) et Colomb (2012), les méthodes pédagogiques universitaires devraient comprendre des pratiques et une communication ayant une congruence avec les cultures autochtones, introduire et valoriser des concepts de ces cultures, ainsi que présenter et mettre de l'avant la contribution présente et historique des peuples autochtones à la société contemporaine. En ce sens, plusieurs étudiants autochtones désireraient que le corps enseignant soit davantage sensibilisé à leurs réalités et à leurs besoins pédagogiques, et qu'il fasse preuve de flexibilité (Adelman et al., 2013; Dufour, 2015; Irwin et Hepplestone, 2012; Joncas, 2013, 2018; Lefevre-Radelli, 2019; Milne et al., 2016; Rodon et Tremblay, 2007; Shield, 2004; Wanner et Palmer, 2015). Pour leur part, Fenelon et LeBeau (2005) recommandent de mettre en place une pédagogie interactive et axée sur le territoire et les récits traditionnels (p. ex., utiliser des histoires, des métaphores de chasse) (Colomb, 2012).

Dans le contexte actuel, plusieurs Autochtones perçoivent l'éducation postsecondaire comme un milieu intégrant et perpétuant une forme d'assimilation qui s'inscrit dans le néocolonialisme, puisque les établissements postsecondaires accordent très peu d'importance au bagage culturel, aux traditions et aux valeurs autochtones (Langevin, 2006; Malatest, 2002; Mareschal et Denault, 2020; Papillon et Cosentino, 2004; Robert-Careau, 2019; Savard, 2010). Il s'avère également que le corps enseignant ignore généralement la dimension spirituelle ${ }^{3}$ des étudiants (Miller et al., 2005) et que les systèmes d'éducation traditionnels sont principalement axés sur la performance

2 Ce concept est présenté dans le cadre conceptuel.

3 L'aspect spirituel ne réfère pas à une croyance religieuse ou à un système de croyances objectives, mais plutôt à une vision singulière que les individus ont de leur relation avec ce qui les entoure (Curwen Doige, 2003). 
scolaire des étudiants et non pas sur leur développement général (Lee et al., 2014; Ricci et Pritscher, 2015). À cet effet, Moldoveanu et Campeau (2016) affirment que la scolarisation actuelle « imposée aux communautés autochtones s'inscrit plutôt dans un paradigme d'échec » (p. 198) et agit négativement sur la confiance en soi et la motivation des étudiants autochtones.

\section{Les objectifs de recherche}

Dans l'état actuel des connaissances, plusieurs recherches ont étudié les parcours de vie des étudiants universitaires autochtones (Bailey, 2016; Cazin, 2005; Loiselle et Legault, 2010; Rodon et Tremblay, 2007; Walton et al., 2020). Il s'avère toutefois que les pratiques enseignantes universitaires sont peu connues (Duguet et Morlaix, 2012; Michaut, 2012; Romainville et Michaut, 2012), notamment celles en contexte de classe incluant ou constituée uniquement d'étudiants autochtones. Les pédagogies utilisées par le corps enseignant constituant des facteurs favorisant la rétention et la réussite des étudiants universitaires (Fontaine et Peters, 2012), il s'avère pertinent de savoir : quelles pratiques sont mises en œuvre par le corps enseignant en contexte de classe autochtone ? Deux objectifs émergent de cette question : 1) identifier les modèles et les méthodes pédagogiques utilisés en contexte de classe autochtone; et 2) identifier les pratiques enseignantes déclarées visant à favoriser les apprentissages à caractère holistique chez les étudiants autochtones universitaires.

\section{Cadre conceptuel}

\section{Les pratiques enseignantes}

Cette recherche s'intéresse aux pratiques enseignantes des professeurs et des chargés de cours universitaires en contexte de classe incluant ou constituée uniquement d'étudiants autochtones et visant à favoriser leur réussite éducative. Selon Altet (2002), les pratiques enseignantes constituent des activités professionnelles intériorisées de façon singulière par les enseignants et appliquées dans des contextes de classes hétérogènes. Ces pratiques sont construites et orientées vers des objectifs et des normes d'un corps professionnel, 
mais tiennent aussi compte de plusieurs dimensions (sociale, psychologique, didactique, pédagogique, épistémique) permettant à l'enseignant de s'adapter aux différentes situations, souvent imprévisibles, en présence d'étudiants (Altet, 2002; Bressoux, 2001). Les pratiques enseignantes englobent ainsi l'ensemble du savoir-faire professionnel, comprenant notamment le temps en présence des étudiants, la préparation des cours et les rencontres entre les professionnels (Bru et Maurice, 2001; de Saint-André et al., 2010; Marcel, 2009). Par ailleurs, ces pratiques englobent les méthodes utilisées par le corps enseignant qui s'inscrivent dans un modèle pédagogique auquel celui-ci peut se référer concernant ses actes professionnels (Morandi et La Borderie, 2001).

\section{Les modèles et les méthodes pédagogiques}

Pour analyser les pratiques enseignantes, il s'avère pertinent d'identifier quels modèles pédagogiques sont adoptés par le corps enseignant, notamment en raison du fait que les étudiants autochtones perçoivent la pédagogie magistrocentrée comme eurocentrée (Colomb, 2012) et que l'utilisation d'une pédagogie pédocentrée est davantage propice à l'acquisition de nouveaux apprentissages et de nouvelles compétences chez les étudiants universitaires (Duguet et Morlaix, 2012; Goodman et al., 2015; Mostrom et Blumberg, 2012; Troop et al., 2015; Wanner et Palmer, 2015). Ces deux modèles pédagogiques, magistrocentré et pédocentré, cohabitent dans les établissements d'éducation universitaire. Dans le modèle magistrocentré, l'enseignant est perçu tel un expert de sa discipline qui doit transmettre son savoir théorique et qui a la responsabilité des apprentissages de ses étudiants (Demougeot-Lebel et Perret, 2010; Frenay, 2006; Lison et Jutras, 2014). Les méthodes pédagogiques dites « traditionnelles » s'inscrivent dans le modèle magistrocentré, comme l'enseignement magistral qui correspond à des « conférences monologues » ou à des « monologues expressifs » (Duguet et Morlaix, 2012). Dans le modèle pédocentré, l'enseignant est considéré comme un accompagnateur qui cherche à favoriser les interactions et à laisser les étudiants devenir maitres de leurs apprentissages (Demougeot-Lebel et Perret, 2010; Frenay, 2006; Lison et Jutras, 2014). Les méthodes pédagogiques dites « innovantes »s'inscrivent dans le modèle pédocentré et englobent « tout ce qui ne relève pas de l'enseignement magistral » (Béchard et Pelletier, 2004, p. 50), comme la méthode des cas, l'apprentissage par problème ou 
par projet (Béchard et Pelletier, 2004). Il n'en demeure pas moins que ces méthodes s'inscrivent dans la tradition occidentale/eurocentrique.

L'utilisation d'une approche favorisant des apprentissages à caractère holistique est grandement recommandée en contexte de classe autochtone (Anuik et Gillies, 2012; Borrows, 2016; Colomb, 2012; Price et Burtch, 2010). Des apprentissages à caractère holistique permettent à l'étudiant de se développer et de trouver un équilibre sur les plans intellectuel, émotionnel, physique et spirituel (Anuik et Gillies, 2012; Bousquet, 2012; Colomb, 2012; Mayes et al., 2016; Miller et al., 2005). Pour susciter de tels apprentissages, le corps enseignant doit adapter le climat de classe aux étudiants tout en laissant une place importante à leurs expériences vécues au sein de leur environnement (Fuentes et Reyes, 2019). Cette expérience doit mener l'étudiant à analyser, dans une perspective globale et une vision intégrée, les éléments du cours qui lui sont présentés, tout en lui accordant la possibilité d'analyser sa relation personnelle avec l'objet d'étude (Colomb, 2012; Fenelon et LeBeau, 2005). La valeur temporelle est essentielle, car ces apprentissages doivent tenir compte de l'interrelation entre le passé, le présent et le futur de l'individu, et demandent davantage de temps qu'une approche traditionnelle qui perçoit l'apprentissage de façon séquentielle, linéaire et hiérarchique (Colomb, 2012). La pédagogie expérientielle est régulièrement perçue comme favorable à l'introduction d'apprentissages holistiques (Colomb, 2012; Curtis et al., 2015; Loiselle et Legault, 2010; Moldoveanu et Campeau, 2016; White, 2001).

\section{Méthode}

Une approche qualitative de type phénoménologique a permis d'examiner les pratiques enseignantes des participants à partir de leur discours et de leurs perceptions (AubinAuger et al., 2008; Mucchielli, 2009; Paillé et Mucchielli, 2003). Cette approche phénoménologique, privilégiant la collecte et l'analyse de données expérientielles, a fourni des informations significatives et propres au vécu des participants, tout en évitant de s'insérer dans un mécanisme explicatif (Mucchielli, 2009; Paillé et Mucchielli, 2003; Savoie-Zajc, 2006). 


\section{Les participants}

Un échantillonnage par homogénéisation a été utilisé pour recruter les participants (Pires, 1997). Cette méthode consiste à déterminer les caractéristiques et les rapports socioculturels établissant l'homogénéisation de la population à l'étude (Fortin et Gagnon, 2016; Pires, 1997). Les critères d'homogénéisation retenus s'appuient sur la Classification nationale des professions de Statistique Canada (2012) et sur le choix d'un établissement universitaire. La Classification nationale des professions a permis d'établir quels étaient les professionnels ayant à s'acquitter de l'enseignement universitaire : 1) les professeurs disposant d'un doctorat dans leur domaine de spécialisation, et 2) les chargés de cours ayant au minimum un diplôme de deuxième cycle. Ensuite, un établissement universitaire permettant d'étudier un contexte de classe autochtone a été choisi, soit l'Université du Québec en Abitibi-Témiscamingue ${ }^{4}$ (UQAT). Ce choix repose notamment sur le temps et les couts inhérents à la recherche dans le cadre d'un projet de maitrise. Bien qu'il s'agisse d'une contrainte, la pertinence de ce lieu d'étude se justifie par sa proximité et ses relations avec des communautés autochtones. En effet, le territoire desservi par cette université comprend notamment sept communautés algonquines (Thibeault, 2015) pour lesquelles elle offre des cours en français et en anglais afin de répondre à leurs besoins. De plus, cet établissement contient l'École d'études autochtones qui offre plusieurs programmes destinés spécifiquement aux autochtones de premier et de deuxième cycle ${ }^{5}$. Ainsi, les facteurs d'inclusion étaient : 1) être professeur ou chargé de cours en activité à l'UQAT, et 2) avoir enseigné dans une classe incluant ou constituée uniquement d'étudiants autochtones.

L'échantillonnage par homogénéisation vise aussi une diversification interne au sein du groupe homogène, c'est-à-dire que le chercheur doit choisir des participants qui présentent différentes caractéristiques (p. ex., âge, genre, années d'expérience, titre de l'employé et département d'enseignement) (Pires, 1997). Au total, l'échantillon comptait six professeurs et quatre chargés de cours (6 hommes et 4 femmes) enseignant dans cinq programmes : études autochtones, développement humain, éducation, gestion ainsi que

4 L'UQAT dessert un territoire éloigné des grands centres urbains et rejoint plus de 6300 étudiants annuellement. Cet établissement compte environ 385 professeurs et chargés de cours.

5 Il n'est pas possible de savoir combien d'étudiants autochtones cheminent à l'UQAT, car cet établissement n'emploie pas de stratégie d'identification systématique lors de l'inscription. 
création et nouveaux médias. Les participants avaient de 3 et 14 années d'expérience en enseignement universitaire et un seul d'entre eux avait déjà suivi une formation universitaire en enseignement postsecondaire, laquelle ne concernait pas l'enseignement en contexte de classe autochtone. Aucun des participants n'était autochtone et seulement trois professeurs avaient présenté des cours universitaires à des classes constituées uniquement d'étudiants autochtones.

Pour recruter les participants, les secrétariats de l'École d'études autochtones, du Service Premiers Peuples de l'UQAT et de chaque unité d'enseignement et de recherche de l'UQAT ont envoyé un courriel d'invitation à tout le corps enseignant. Le recrutement des participants a par ailleurs constitué un défi ${ }^{6}$ puisque, malgré les stratégies de recrutement répétées pour augmenter le nombre de participants, seules dix personnes ont manifesté leur intérêt et ont finalement participé à l'étude. Par conséquent, il n'a pas été possible d'assurer, comme prévu, une diversification interne.

\section{La collecte et l'analyse des données ${ }^{7}$}

Les données ont été collectées d'octobre 2017 à octobre 2018 au moyen d'entrevues semi-dirigées, lesquelles favorisent une compréhension des phénomènes par le point de vue et le sens que les participants ont de leur réalité (Boutin, 2000; Savoie-Zajc, 2010). Les entrevues, d'une durée de 60 à 90 minutes, se sont déroulées sur le lieu de travail des participants et leur discours a été recueilli à l'aide d'un enregistreur vocal. Le Tableau 1 présente les différents thèmes abordés ${ }^{8}$.

6 Les refus obtenus découlent en majorité du fait que les participants potentiels n'avaient pas conscience de la présence d'étudiants autochtones au sein de leur groupe.

7 Cette recherche a été réalisée dans le cadre d'études de deuxième cycle. C'est l'étudiant-chercheur (premier auteur de cet article) qui a réalisé la collecte et l'analyse des données, et ce, sous la supervision de son directeur de recherche (le second auteur de cet article).

8 Les résultats présentés dans ce texte sont issus d'un projet de maitrise et ceux-ci ne couvrent que deux des quatre objectifs de la recherche. Le guide d'entrevue était donc composé d'autres thèmes qui ne sont pas présentés dans cet article. 


\section{Tableau 1}

Thèmes de l'entrevue

\begin{tabular}{|c|c|c|}
\hline & Thèmes & Sous-thèmes \\
\hline Objectif 1 & $\begin{array}{c}\text { Connaissances au regard } \\
\text { des enseignements }\end{array}$ & $\begin{array}{c}\text { Connaissances pédagogiques } \\
\text { Habiletés pédagogiques } \\
\text { Connaissances sur la culture autochtone }\end{array}$ \\
\hline Objectif 2 & Pratiques enseignantes & $\begin{array}{c}\text { Déroulement des cours } \\
\text { Rôles des étudiants dans le cours } \\
\text { Techniques utilisées en classe } \\
\end{array}$ \\
\hline
\end{tabular}

Les entrevues ont été transcrites, puis une analyse thématique a été menée pour « dégager quels thèmes sont présents, avec quelle importance, associés de quelle façon, etc. » (Paillé et Mucchielli, 2003, p. 145). Enfin, les extraits encodés manuellement dans le logiciel QSR NVivo 10 ont été soumis à un examen discursif qui consiste à mettre en relation des thèmes avec le cadre conceptuel de la recherche et/ou les écrits scientifiques (Fortin et Gagnon, 2016). Le type de codage utilisé était mixte, c'est-à-dire que certains thèmes étaient prédéfinis par le cadre conceptuel, mais que les thèmes analysés étaient aussi ouverts à des modifications et des ajouts (Van der Maren, 2004).

Pour répondre aux critères de scientificité (Lincoln et Guba, 1985), plusieurs stratégies ont été mises en œuvre. D'abord, la réplication par étape (stepwise replication), effectuée par les deux auteurs de cet article, ainsi que la saturation théorique ont été utilisées pour favoriser la fiabilité des résultats (Lincoln et Guba, 1985; Pires, 1997). Ensuite, le recours à la méthode de l'audit a permis d'assurer la validation, c'est-àdire que le directeur de recherche a joué le rôle d'expert en vérifiant la valeur des interprétations de l'étudiant-chercheur. Enfin, la transférabilité des résultats est possible puisque le contexte de l'étude ainsi que les considérations méthodologiques sont décrits dans le mémoire de l'étudiant (voir Gingras-Lacroix, 2019). La crédibilité des résultats constitue néanmoins une limite puisqu'aucune méthode de triangulation ou de vérification par les participants n'a été utilisée (Lincoln et Guba, 1985; Savoie-Zajc, 2009) 


\section{Résultats}

Cette section présente les pratiques enseignantes dégagées du discours des participants. La première section décrit les modèles et les méthodes pédagogiques utilisés en contexte de classe ayant au moins un étudiant autochtone. La deuxième section aborde plus précisément les résultats concernant la préoccupation des participants au regard des apprentissages à caractère holistique. Enfin, la troisième section présente les pratiques pédagogiques que les participants ont affirmé mettre en œuvre dans le but de favoriser la réussite éducative des étudiants autochtones.

\section{Les modèles et les méthodes pédagogiques utilisés en contexte de classe autochtone}

Il s'avère que tous les participants adoptent les deux modèles pédagogiques. En effet, le choix du modèle pédagogique adopté varie selon : 1) les objectifs du cours (axés sur la théorie ou la pratique); 2) le mode du cours (en présentiel ou par visioconférence); et 3) le nombre d'étudiants. À cet effet, tous les participants ont mentionné qu'avoir des « groupes de taille humaine » (participant 3) fait en sorte qu'il devient « pédagogiquement plus facile d'adapter ses pratiques que dans une classe de 40-45 étudiants » (participant 7). Il n’y a cependant pas de consensus en ce qui concerne la pédagogie qui serait la plus appropriée aux étudiants autochtones. Six participants soulignent que ces étudiants préfèrent une pédagogie pédocentrée : « pour moi, c'est une pédagogie qui regroupe tout le monde qui désire apprendre, et encore plus chez les étudiants autochtones » (participant 10). Pour quatre autres participants, ce n'est pas le fait que les pratiques soient axées sur des méthodes traditionnelles (magistrocentrées) ou innovantes (pédocentrées) qui influence la réussite des étudiants autochtones, mais davantage le fait que ces dernières soient axées sur ce qui est concret. Par exemple, le participant 8 déclare : «Souvent, je dis aux étudiants : Ça, c'est en théorie... mais dans la vraie vie maintenant, ce n'est pas toujours comme ça que ça se passe !». Ainsi, selon neuf participants, il n'y aurait pas une pédagogie propre aux étudiants autochtones : «j'en ai presque à chaque année des étudiants autochtones, puis c'est la même façon de faire qu'avec tout le monde. Je ne fais pas une différence » (participant 9). 
Parmi les méthodes pédagogiques recensées dans le discours des participants, il a été possible de constater qu'ils ont tous recours à la présentation magistrale du contenu théorique. Le coenseignement est une méthode qui a été mise en place uniquement par le participant 5. Ce dernier explique que cette méthode lui demande « d'accompagner » les étudiants qui doivent «s'approprier la matière » afin d'être « en mesure de la redonner aux autres ». Son rôle consiste à « valider » ce qu'ils présentent, à « corriger » et à « ajuster s'il y a des choses qui ne sont pas bien comprises » (participant 5).

L'enseignement par projet est une autre méthode pédagogique utilisée par quatre participants. Celle-ci n'est toutefois pas mise en place de la même façon par ces participants. Dans le cas du participant 1 , « il y a une première session où on donne les bases théoriques et techniques, puis la deuxième session où on passe en mode projet par rapport à une thématique ». Ce participant offre une liberté à ses étudiants quant au choix du projet : «Ils doivent trouver un projet à faire et puis c'est sûr que ce projet-là, ils le font à partir de ce qu'ils vivent, d'où est-ce qu'ils veulent aller ». Le participant 5 , quant à lui, propose à ses étudiants de réaliser un « projet individuel, mais qui va faire partie d'un projet collectif $»$. Il explique que cette méthode pédagogique touche, selon lui, à la fois « la collaboration et l'individu » puisqu' « il faut que l'individu ait un bagage pour être vraiment à même de collaborer avec les autres puis d'apporter quelque chose aux autres ». Le participant 4 a pour sa part moins aimé son expérience d'enseignement par projet puisqu'il considère que cela s'est révélé « un échec monumental ». Dans ce cas, le projet était proposé à un groupe composé uniquement d'étudiants autochtones et consistait en un «travail à produire » qui s'étalait sur toute la session. Le participant raconte comment cela s'est passé :

J'essayais de les appuyer, j'essayais de les suivre... mais ils voyaient ça comme une grande période de temps libre avec un travail à remettre à la fin. J'ai eu beau tout décortiquer le travail qu'ils avaient à faire : « ok, cette semaine vous devez appeler pour rencontrer des gens... » Ça n’a pas du tout marché. (Participant 4)

Une autre méthode pédagogique utilisée par cinq des participants est la mise en situation. Pour les participants 2, 7 et 10, ces mises en situation sont vécues sous forme de jeux de rôle. Par exemple, l'un d'eux propose à ses étudiants des situations fictives et transforme la salle de classe en « laboratoire d'expérimentation d'intervention sociale ». Pour les participants 6 et 8 , les mises en situation se réalisent plutôt sous forme écrite. En 
ce sens, le participant 6 propose à ses étudiants de se plonger dans une mise en situation dans le cadre d'un travail écrit à rédiger :

Moi, c'est : « trouve-moi une situation dans la vraie vie puis interviens. Travaille là-dessus. Ou invente un "business" » Ça, j'aime bien ce genre de projet là. Quelqu'un a vraiment une liberté totale. Il choisit le produit que ça lui tente et il met une stratégie en œuvre, sur le papier. (Participant 6)

Une autre méthode pédagogique déclarée par deux participants consiste à partir de la pratique (p. ex., le vécu et les expériences des étudiants) pour aller vers la théorie. Le participant 2 qualifie cette méthode d' " approche inductive » dans laquelle, « à partir de choses qu'on fait, on essaie d'en tirer des généralisations, des idées ». Le participant 7 la qualifie quant à lui de « pédagogie inversée ». L'extrait suivant illustre dans quel contexte ce participant y a recours :

À l'UQAT, j'ai déjà enseigné à un groupe d'adultes que ça faisait 10-15 ans qu'ils étaient sur le terrain. Ils avaient des techniques, ils faisaient leur baccalauréat. On fonctionnait à l'inverse. C'est une pédagogie inversée quand les étudiants ont beaucoup de pratique : on y va par les éléments concrets qu'ils ont vécus, on les décortique, on les analyse. Puis, après ça, on les rattache à des éléments théoriques.

Il est néanmoins important de souligner que les participants ont affirmé qu'ils choisissaient les méthodes pédagogiques utilisées indépendamment de la présence ou non d'étudiants autochtones dans le groupe classe : « Je dis autochtone - allochtone, mais moi, je ne fais pas tant que ça une grosse distinction à part l'aspect culturel » (participant 3); « Moi j'en ai presque à chaque année (des étudiants autochtones), puis c'est la même façon de faire qu'avec tout le monde » (participant 9). Ce choix est parfois influencé par le nombre d'étudiants autochtones dans le groupe : « Mes pratiques sont sensiblement la même chose parce que les étudiants autochtones sont un faible pourcentage d'étudiants dans mes cours. [...] Si j'ai 50 personnes et que j'ai une personne autochtone, jusqu'où je dois aller dans l'adaptation » (participant 7). Néanmoins, aucune tendance de ce choix de pratique n'a pu être dégagée en fonction de la proportion d'étudiants autochtones présents dans le groupe ou de l'endroit dans lequel avait lieu le cours (sur le campus ou sur la réserve autochtone). 


\section{Les apprentissages à caractère holistique}

En ce qui concerne les apprentissages à caractère holistique, soit la possibilité de se développer sur les plans physique, cognitif, émotionnel et spirituel, la moitié des participants affirment être ouverts au fait que leurs étudiants autochtones les intègrent dans leurs travaux et leurs productions, dans la mesure où le travail demandé répond aux critères d'évaluation. En ce sens, selon le participant 8 , la création d'un portfolio par les étudiants est un moyen pour eux de toucher certains éléments spirituels : « il y en a qui vont me parler de leurs croyances [...]. Ce serait plus là que je te dirais qu'on irait toucher l'expérience spirituelle».

L'un des travaux proposés par un participant enseignant dans le domaine de l'audiovisuel offre également aux étudiants la possibilité d'acquérir des apprentissages qui ont une portée holistique. En effet, les étudiants allochtones et autochtones doivent ici produire une vidéo dont l'évaluation porte sur la technique, la lumière, la colorimétrie, la musique, etc. Or, les étudiants sont libres dans leur création. L'extrait suivant en est d'ailleurs un bon exemple :

Je viens de penser à une autre personne qui était autochtone, à qui j'enseignais. Il y avait un processus de guérison dans son travail. Un processus de guérison intérieure. Un processus de guérison et de transformation de soi. Il y avait, dans tous les cas, une volonté de se servir de l'art comme un mode de transformation, de métamorphose, comme d'éclosion. Pas de renaissance, mais comme un serpent qui change de mue. (Participant 1)

Toutefois, dans le discours des participants, aucune méthode pédagogique visant explicitement des apprentissages holistiques n'a pu être identifiée. En effet, comme le souligne le participant $8:$ «On reste quand même beaucoup dans l'intellectuel, le cognitif ». Le participant 9 indique pour sa part : « Je n'utilise pas de pratiques pédagogiques holistiques. Parfois, je présente des outils qui touchent des approches holistiques, mais on ne va pas vivre la tente tremblante dans la classe, par exemple ». Cinq participants affirment néanmoins qu'ils aimeraient inclure davantage des apprentissages à caractère holistique au sein de leurs pratiques : « Je pense que j'y touche un petit peu (apprentissage holistique), mais je pense que ça pourrait être plus poussé » (participant 8). Néanmoins, comme le mentionne le participant 9, ajouter de tels 
apprentissages s'avère difficile puisque : «c'est tellement rigide la culture universitaire que je pense que c'est mieux de faire des pas en classe dans la relation et les outils ». Aucune stratégie n'a donc été mentionnée par les participants afin d'ajouter des activités favorisant des apprentissages à caractère holistique.

\section{Les pratiques enseignantes visant à favoriser la réussite des étudiants autochtones}

Les discussions concernant les pratiques enseignantes ont permis de faire ressortir des éléments qui, selon les participants, favorisent la réussite des étudiants autochtones. Ainsi, établir des relations positives basées sur le respect, l'écoute, l'ouverture et la flexibilité sont des pratiques importantes à mettre en œuvre selon les participants, de même qu'adapter le contenu du cours aux réalités et au vécu des étudiants autochtones.

\section{Interagir en faisant preuve d'écoute, de flexibilité, d'ouverture et de respect.}

Dans le discours des participants, quatre attitudes, qui favorisent selon eux les apprentissages des étudiants autochtones, ont été dégagées, soit : l'écoute, la flexibilité, l'ouverture et le respect, notamment envers la notion de silence, les savoirs autochtones et le rythme d'apprentissage des étudiants. Huit participants considèrent que le fait d'être à l'écoute de leurs étudiants est une attitude importante à développer, et ce, particulièrement auprès des étudiants autochtones. À cet effet, certains participants expliquent qu'être à leur écoute permet de connaitre leur vécu, notamment en ce qui concerne « le départ de leur communauté, difficile pour certains, mais extraordinaire pour d'autres » (participant 4), ou encore les situations particulières auxquelles certains ont été confrontés, comme «l'effondrement des relations familiales dans leur communauté, la pauvreté aigüe dans laquelle ils vivent, les parcours scolaires atypiques qu'ils ont » (participant 2).

Être à l'écoute afin de connaitre le vécu des étudiants autochtones est d'ailleurs lié à une autre attitude qui a été identifiée dans le discours de tous les participants : la flexibilité. Entre autres, le participant 3 explique qu'il adapte le calendrier des cours en fonction des demandes faites par ses étudiants autochtones, par exemple, en déplaçant un cours qui est prévu le même jour qu'un pow-wow ou qu'un tournoi de hockey. Deux participants ont également mentionné que la notion de temps pour plusieurs étudiants 
autochtones est différente de celle qui est attendue dans les universités et que cela demande donc une certaine flexibilité à l'égard du délai de remise des travaux.

L'ouverture est aussi une attitude à développer selon tous les participants. Cette dernière les amène parfois à revoir leurs méthodes de travail, ainsi que leur conception du milieu universitaire. En ce sens, deux participants constatent que l'écriture, notamment celle des travaux et des mémoires, diffère chez les étudiants autochtones. Par exemple, deux participants font preuve d'ouverture envers la façon qu'ont leurs étudiants autochtones de présenter leurs travaux qui sont « plus biographiques que théoriques, plus axés sur l'expérience personnelle, sur le récit » (participant 2). Le participant 2 a fait ce constat alors qu'il encadrait un étudiant autochtone aux cycles supérieurs :

Quand il fait ses résultats de recherche, il ne présente pas ça comme nous autres : il présente ça sous la forme d'un récit. De l'avant, du passé, de l'est, de l'ouest, de la roue médicinale. Puis chaque partie du récit, il base ça sur le récit d'une personne qu'il a rencontrée pendant son stage d'essai. Donc, on va faire un récit, ça va être le bison, la perdrix, la loutre et le renard dans les récits qu'il va faire. Puis quand tu lis les mémoires de maitrise d'étudiants amérindiens au travers du Canada, tu vas te rendre compte des grands récits et que la structure de pensées n'est pas la même.

Faire preuve de respect, selon deux participants, implique d'être en mesure de « comprendre et de décrypter» (participant 5) les silences qui se produisent en contexte de classe avec des étudiants autochtones puisque « le silence est important pour les étudiants autochtones » (participant 7). Le participant 5 explique que, pour ces derniers, le concept de silence «n'est pas nécessairement un synonyme de passivité ou de désintérêt ». En ce sens, pour le participant 7, le silence est pour ces étudiants un temps pour « réfléchir » et « regarder » puisqu' « habituellement, dans nos cours, on va beaucoup trop vite pour eux ». Il ajoute qu'il faut laisser aux étudiants autochtones « le temps d'intégrer la matière » et souligne qu'ils ont besoin de voir comment ils peuvent la transmettre et l'adapter au sein de leur communauté.

Tous les participants affirment qu'il est essentiel de respecter les savoirs autochtones. Cette attitude signifie pour eux qu'ils doivent reconnaitre les différences et les richesses liées aux cultures autochtones, mais aussi reconnaitre les savoirs et les compétences de ces étudiants. 
Le participant 5 considère que « c'est important d'avoir quand même une petite connaissance de qui tu as en face de toi. Sinon, on reste dans une dimension encore une fois d'étrangeté, puis de dominant/dominé, puis on ne crée rien ». Le participant 7 souligne que « s'il y a des étudiants autochtones qui voient qu'on a une préoccupation par rapport à leur culture, bien c'est un respect. Et moi, je me dis qu'un étudiant qui sent qu'il est respecté par le professeur, il risque plus d'être partie prenante bien sûr ». Ce respect favorise ainsi, selon les participants, l'établissement d'un lien de confiance avec les étudiants autochtones ainsi que leur participation en classe.

\section{Adapter le contenu du cours aux réalités et au vécu des étudiants autochtones.}

Les résultats révèlent que les participants ne choisissent pas leurs méthodes pédagogiques en fonction du contexte de classe autochtone, et ce, peu importe la proportion d'étudiants autochtones dans le groupe. Les discours analysés permettent toutefois de constater que les participants considèrent qu'il est important d'adapter le contenu du cours aux réalités et au vécu des étudiants autochtones. En effet, tous les participants rencontrés considèrent que ces étudiants cheminent dans un programme allochtone qui n'est pas toujours adapté à leur culture et à leur réalité. À cet effet, le participant 7 explique que, selon lui, les interventions sociales enseignées à l'université sont centrées sur la culture «blanche» et que « ce n'est pas toujours adapté à leur culture. Loin de là. C'est loin d'être adapté et ce n'est pas toujours évident de l'adapter ». Ainsi, cela représente un défi pour le corps enseignant rencontré puisque, dans de telles situations, les étudiants «n'ont rien à s'accrocher » (participant 3 ). De plus, comme le souligne le participant $2:$ «t tu peux leur enseigner des cours super théoriques, mais s'ils n'y voient pas d'utilités, ils décrochent bien vite. Donc, ils se retrouvent démunis sur des plans théoriques quelquefois ».

Une pratique utilisée par tous les participants consiste donc à bonifier les contenus avec des exemples qui accordent une importance aux territoires sur lesquels évoluent les communautés autochtones. Ces exemples peuvent notamment provenir du vécu et des connaissances des étudiants autochtones eux-mêmes : « J'adapte en me servant de l'expérience de mes étudiants pour enrichir le cours » (participant 5). Selon les participants, l'utilisation de l'expérience des étudiants autochtones permet à ces derniers de mieux comprendre le monde dans lequel ils évoluent et de faire des liens avec la théorie vue en classe : «J'essaie de rattacher la théorie à des faits, des situations, des problèmes qu'ils ont rencontrés parce que, des fois, ils trouvent ça dur et ils ont de la 
difficulté à comprendre la théorie » (participant 9). Lier le contenu du cours aux réalités et aux cultures autochtones n'est cependant pas toujours simple lorsqu'il y a peu d'étudiants autochtones dans le groupe. En ce sens, l'un des participants mentionne que, pour lui, cette adaptation se fait davantage lors de rencontres qui se déroulent en dehors des heures de cours afin de «s'assurer de ce que les étudiants ont compris, de quelle façon ça pourrait être traduit et comment ça pourrait être vulgarisé pour travailler au niveau de leur communauté » (participant 7). Or, il reconnait que cela implique une certaine volonté personnelle en raison de l'investissement de temps qui y est rattaché : «J'en ai passé des heures, moi, avec des étudiants autochtones en dehors de mes cours ! Parce que j'en ai fait une volonté personnelle. [...] Souvent, on donne quasiment un deuxième cours » (participant 7).

\section{Discussion}

Dans l'état actuel des connaissances, peu de données sont disponibles concernant les pratiques du corps enseignant universitaire visant à favoriser la réussite éducative des étudiants autochtones. Cette recherche s'est ainsi penchée sur les modèles et les méthodes pédagogiques utilisés à l'université en contexte de classe incluant ou constituée uniquement d'étudiants autochtones, ainsi que sur les pratiques enseignantes visant à favoriser les apprentissages à caractère holistique chez ces étudiants. Cette section présente une discussion concernant les principaux résultats dégagés de cette étude.

Cette recherche a permis de constater que les participants ne considèrent pas qu'il existe un modèle ou des méthodes pédagogiques qui sont plus propices aux apprentissages des étudiants autochtones. Par conséquent, les méthodes pédagogiques utilisées par les participants correspondent à la fois au modèle magistrocentré et au modèle pédocentré. Par ailleurs, l'ensemble des méthodes pédagogiques identifiées s'inscrivent davantage dans une perspective eurocentrique. Ce résultat pourrait s'expliquer par le fait que les participants affirment ne pas choisir leurs méthodes pédagogiques en fonction de l'origine culturelle de leurs étudiants, mais plutôt en fonction de leur désir de répondre aux besoins des différents étudiants du groupe. En s'appuyant sur les résultats d'une revue de la littérature australienne (Lloyd et al., 2015, p. 13), il est possible d'affirmer que cette pratique enseignante est pertinente dans la 
mesure où il ne faut pas souscrire à une vision réductionniste qui implique l'existence d'une pédagogie « efficace » uniforme pour les étudiants autochtones ou une pédagogie spécifique à la culture qui crée un cadre binaire « à deux races ». Toutefois, il est également erroné de considérer qu'une approche pédagogique peut être appliquée à tous les étudiants, autochtones ou allochtones, puisqu'un enseignement efficace repose sur le développement du plein potentiel éducatif de chaque étudiant (s'adapter à ses connaissances, ses compétences, ses valeurs, ses normes, ses ressources et son histoire) (Castagno et Brayboy, 2008; Lloyd et al., 2015). Par conséquent, pour favoriser la réussite éducative des étudiants autochtones, le corps enseignant universitaire gagnerait à adopter des méthodes pédagogiques qui visent des apprentissages à caractère holistique chez les étudiants autochtones (Borrows, 2016; Price et Burtch, 2010).

À ce sujet, les résultats de cette recherche dévoilent que plusieurs participants utilisent des méthodes relatives à une pédagogie expérientielle, comme les jeux de rôle, les mises en situation et la création de projets. Or, si ces méthodes permettent le développement global de l'étudiant, favorisent son implication consciente et lui laissent la possibilité d'apprendre selon son vécu et son ressenti (Côté, 1998; Kold, 1986; Moldoveanu et Campeau, 2016), ces méthodes ne sont pas choisies par les participants dans le but de favoriser intentionnellement des apprentissages à caractère holistique. Ce constat pourrait s'expliquer par le fait que le corps enseignant n'a pas suffisamment de connaissances ou de formation au sujet de la pédagogie universitaire en général (Fontaine et Peters, 2012; Lison, 2011; Walder, 2015), et plus spécifiquement sur 1'histoire et les expériences des populations autochtones ainsi que sur les approches pédagogiques holistiques (Blimkie et al., 2014; Nardozi et al., 2014), pour être en mesure de transformer ses pratiques. En effet, lors des entrevues, la grande majorité des participants ont mentionné ne pas avoir reçu de formation relative à l'enseignement universitaire. Certains participants de cette recherche ont tout de même affirmé vouloir adopter des méthodes pédagogiques favorisant des apprentissages à caractère holistique. Le contexte universitaire présente néanmoins des obstacles à ce changement de pratiques, comme le manque de temps à consacrer à la préparation des cours (de Hosson et al., 2014; Walder, 2015), le manque de collaboration entre les membres du corps enseignant découlant d'une perception que l'enseignement est une tâche individuelle (Töytäri et al., 2017) ainsi que la culture universitaire axée sur la performance scolaire des étudiants et non sur leur développement holistique (Lee et al., 2014; Ricci et Pritscher, 2015; Sanford et al., 2012). 
Il est également possible que puisque le corps enseignant n'est pas informé à l'avance de la composition de son groupe et qu'il ignore s'il enseignera ou non à des étudiants autochtones, il ne soit pas en mesure de planifier sa pédagogie en conséquence. Une piste de solution pourrait donc être la prise d'initiatives par les universités afin d'assurer l'acquisition de connaissances pédagogiques favorisant des apprentissages à caractère holistique par le corps enseignant.

Cette recherche a également révélé que les participants rencontrés ont conscience de l'aspect eurocentrique de l'enseignement supérieur, comme constaté dans d'autres recherches (Battiste, 2013; Fiddler, 2015), et qu'ils mettent en œuvre des moyens individuels pour tenter d'y remédier. En effet, les participants ont souligné l'importance pour eux de faire preuve d'écoute, de flexibilité, d'ouverture et de respect envers les étudiants autochtones afin de favoriser leur réussite éducative. Cela rejoint le désir des étudiants autochtones que le corps enseignant soit sensibilisé à leurs besoins pédagogiques et qu'il fasse preuve de flexibilité (Adelman et al., 2013; Dufour, 2015; Irwin et Hepplestone, 2012; Joncas, 2013, 2018; Lefevre-Radelli, 2019; Milne et al., 2016; Rodon et Tremblay, 2007; Shield, 2004; Wanner et Palmer, 2015). De plus, les participants adaptent à différents degrés le contenu de leurs cours en y intégrant des exemples propres aux réalités des communautés autochtones, en laissant place à la discussion sur le vécu et les connaissances des étudiants ainsi qu'en accordant une place aux expériences personnelles de ces derniers, et ce, dans une visée d'inclusion et d'équité. Il s'avère néanmoins qu'une faible proportion d'étudiants autochtones dans le groupe serait un frein à cette adaptation, ce qui ne serait pas étranger à ce que soulèvent Loiselle et Legault (2010) quant au fait que les étudiants autochtones se sentiraient davantage « dans un endroit pertinent » lorsqu'il y a plus de pairs autochtones dans leurs groupes.

\section{Conclusion}

Cette étude met en valeur l'importance de s'intéresser aux pratiques enseignantes mises en œuvre à l'université en contexte de classe incluant ou constituée uniquement d'étudiants autochtones, puisque celles-ci se situent toujours dans une perspective principalement eurocentrique. En effet, bien que le corps enseignant rencontré cherche à adapter ses 
pratiques, il n'est pas question d'une transformation (Kermoal, 2018) visant, par exemple, à utiliser des méthodes favorisant les apprentissages à caractère holistique. Cette étude contribue ainsi à alimenter les réflexions des universités, de leur service de pédagogie universitaire, des organismes hors communauté offrant des services aux Autochtones ainsi que celles des communautés autochtones qui ont une autorité en éducation aux adultes quant à la création d'ententes avec le gouvernement et les établissements d'éducation collégiale et universitaire (Commission de l'éducation, 2007; Fraternité des Indiens du Canada, 1972). En effet, à la lumière des résultats soulevés dans la discussion, ces acteurs pourront se questionner sur les orientations qu'ils désirent mettre de l'avant.

Cette étude présente trois principales limites qu'il importe de considérer dans l'interprétation des résultats. La première concerne le nombre restreint de participants. En ce sens, la collecte de données a pris fin par manque de participants. Ainsi, il est important de mentionner que les résultats obtenus ne représentent pas l'ensemble des pratiques du corps enseignant de l'UQAT en contexte de classe incluant ou constituée uniquement d'étudiants autochtones. La deuxième limite concerne la nature des résultats dégagés dans cette étude. En effet, les pratiques enseignantes qui sont décrites dans cet article représentent l'expérience professionnelle et la perception des participants. À cet effet, étant donné l'utilisation hétérogène des pratiques enseignantes singulières à chacun des professeurs et chargés de cours rencontrés, il est impossible de prétendre que les pratiques utilisées par les participants sont efficientes auprès des étudiants autochtones. Le discours des étudiants n'ayant pas été exploré, il est donc impossible d'affirmer si ces pratiques sont perçues positivement par ces derniers. La troisième limite concerne le fait que les participants n'avaient pas tous la même compréhension de ce qu'étaient des apprentissages à caractère holistique. Pour limiter ce biais, cette notion leur a été brièvement expliquée lors de l'entrevue.

En conclusion, cette étude permet de constater les efforts individuels mis en place par le corps enseignant pour rendre l'enseignement universitaire plus inclusif envers les étudiants autochtones. Elle met également en lumière le chemin qu'il reste à parcourir afin de transformer le milieu universitaire pour que ces changements favorisent la réussite éducative des étudiants autochtones. En ce sens, le rapprochement des peuples en éducation nécessite un dialogue et des transformations profondes qui passent notamment par l'intégration des méthodes d'enseignement et des connaissances autochtones en classe (Johanne, 2020). 


\section{Références}

Adelman, H., Taylor, L. et Nelson, P. (2013). Native American students going to and staying in postsecondary education: An intervention perspective. American Indian Culture and Research Journal, 37(3), 29-56. https://doi.org/10.17953/ aicr.37.3.01130638k210j380

Altet, M. (2002). Une démarche de recherche sur la pratique enseignante : l'analyse plurielle. Revue française de pédagogie, (138), 85-93. https://doi.org/https://doi. org/10.3406/rfp.2002.2866

Anuik, J. et Gillies, C. L. (2012). Indigenous knowledge in post-secondary educators' practices: Nourishing the learning spirit. Canadian Journal of Higher Education, 42(1), 63-79. https://doi.org/https://doi.org/10.47678/cjhe.v42i1.1902

Archambault, H. (2010). Quels sont les facteurs favorisant ou inhibant la réussite éducative des élèves autochtones? First Peoples Child \& Family Review, 5(2), 107-116. https://doi.org/10.7202/1068936ar

Aubin-Auger, I., Mercier, A., Baumann, L., Lehr-Drylewicz, A.-M., Imbert, P., Letrilliart, L. et le GROUM-F. (2008). Introduction à la recherche qualitative. Exercer, 19(84), 142-145. http://www.bichat-larib.com/publications. documents/3446_2008 introduction_RQ Exercer.pdf

Augustus, C. (2015). Knowledge liaisons: Negotiating multiple pedagogies in global indigenous studies courses. Canadian Journal of Higher Education, 45(4), 1-17. https://doi.org/10.47678/cjhe.v45i4.184894

Bailey, K. A. (2016). Racism within the Canadian university: Indigenous students' experiences. Ethnic and Racial Studies, 39(7), 1261-1279. https://doi.org/10.1080 $\underline{\text { 001419870.2015.1081961 }}$

Battiste, M. (2008). The decolonization of Aboriginal education: Dialogue, reflection, and action in Canada. Dans P. R. Dasen et A. Akkari (dir.), Educational theories and practices from the majority world (p. 168-195). SAGE. http://dx.doi. org/10.4135/9788132100683.n8

Battiste, M. (2013). Decolonizing Education: Nourishing the Learning Spirit. Purich Publishing Limited. 
Battiste, M. et Henderson, J. Y. (2009). Naturalizing indigenous knowledge in Eurocentric education. Canadian Journal of Native Education, 32(1), 5-18. https://acurriculumjourney.files.wordpress.com/2014/04/battiste-henderson-2009naturalizing-indigenous-knowledge-in-eurocentric-education.pdf

Béchard, J.-P. et Pelletier, P. (2004). Les universités traditionnelles : à l'heure des innovations pédagogiques. Gestion, 29(1), 48-55. https://doi.org/https://doi. org/10.3917/riges.291.0048

Black, G. L. et Hachkowski, C. (2018). Indigenous learners: what university educators need to know. Journal of Further and Higher Education, 43(8), 1092-1108. https://doi.org/10.1080/0309877x.2018.1450495

Blimkie, M., Vetter, D. et Haig-Brown, C. (2014). Shifting perspectives and practices: Teacher candidates' experiences of a First Nation, Métis and Inuit infusion in mainstream teacher education. Brock Education, 23(2), 47-66.

Borrows, J. (2016). Outsider education: Indigenous law and land-based Learning. Windsor Yearbook of Access to Justice, 33(1), 1-27. https://doi.org/10.22329/ wyaj.v33i1.4807

Bousquet, M.-P. (2012). Êtres libres ou sauvages à civiliser ? L'éducation des jeunes Amérindiens dans les pensionnats indiens au Québec, des années 1950 à 1970. Revue d'histoire de l'enfance « irrégulière ». Le Temps de l'histoire, (14), 162192. https://doi.org/10.4000/rhei.3415

Bousquet, M. (2008). How the university works: Higher education and the low-wage nation. New York University Press.

Boutin, G. (2000). L'entretien de recherche qualitatif. Presses de l'Université du Québec.

Bressoux, P. (2001). Réflexions sur l'effet-maître et l'étude des pratiques enseignantes. Les Dossiers des Sciences de l'Éducation, (5), 35-52. https://doi.org/10.3406/ dsedu.2001.949

Bru, M. et Maurice, J.-F. (dir.). (2001). Les pratiques enseignantes. Contributions plurielles [numéro thématique spécial]. Les Dossiers des Sciences de l'Éducation, (5). https://www.persee.fr/issue/dsedu_1296-2104_2001_num_5_1 
Castagno, A. E. et Brayboy, B. M. J. (2008). Culturally responsive schooling for Indigenous youth: A review of the literature. Review of Educational Research, 78(4), 941-993. https://doi.org/10.3102/0034654308323036

Cazin, A. (2005). Bilan de deux enquêtes auprès d'étudiants autochtones anglophones admis à l'UQAT à un programme à temps plein à l'automne 2004. UQAT, Laboratoire de recherche pour le soutien des communautés. https://www.uqat.ca/ telechargements/info_entites/enq_autochtones.pdf

Colomb, E. (2012). Premières Nations : essai d'une approche holistique en éducation supérieure. Presse de 1'Université du Québec. https://doi.org/10.2307/j. ctv18ph7hr

Commission de l'éducation. (2007). La réussite scolaire des Autochtones. Secrétariat des commissions de l'Assemblée nationale du Québec. https://numerique.banq.qc.ca/ patrimoine/details/52327/1762627

Côté, R. L. (1998). Apprendre : formation expérientielle stratégique. Presses universitaires du Québec.

Curtis, E., Wikaire, E., Kool, B., Honey, M., Kelly, F., Poole, P., Barrow, M., Airini, Ewen, S. et Reid, P. (2015). What helps and hinders indigenous student success in higher education health programmes: a qualitative study using the Critical Incident Technique. Higher Education Research \& Development, 34(3), 486-500. https://doi.org/10.1080/07294360.2014.973378

Curwen Doige, L. A. (2003). A missing link: Between traditional Aboriginal education and the Western system of education. Canadian Journal of Native Education, 27(2), 144-160. http://schoolandsociety.weebly.com/uploads/1/1/5/2/11522132/a missing_link_ _ between traditional_aboriginal_education and the western system of education.pdf

de Hosson, C., Décamp, N., Morand, E. et Robert, A. (2014). Exploration de pratiques pédagogiques déclarées dans l'enseignement supérieur : enquête semiquantitative auprès d'enseignants-chercheurs de physique. Actes des $8 \mathrm{e}$ rencontres de l'ARDIST, Marseille, France. https://hal.archives-ouvertes.fr/ hal-01663287/document 
de Saint-André, M. D., Montésinos-Gelet, I. et Morin, M.-F. (2010). Avantages et limites des approches méthodologiques utilisées pour étudier les pratiques enseignantes. Nouveaux cahiers de la recherche en éducation, 13(2), 159-176. https://doi. org/10.7202/1017288ar

Demougeot-Lebel, J. et Perret, C. (2010). Identifier les conceptions de l'enseignement et de l'apprentissage pour accompagner le développement professionnel des enseignants débutants à l'université. Savoirs, 23(2), 51-72. https://doi.org/https:// doi.org/10.3917/savo.023.0051

Dufour, E. (2015). La sécurité culturelle en tant que moteur de réussite postsecondaire : enquête auprès d'étudiants autochtones de l'Institution Kiuna et des espaces adaptés au sein des établissements allochtones [Mémoire de maitrise, Université de Montréal]. Papyrus. https://papyrus.bib.umontreal.ca/xmlui/handle/1866/13638

Duguet, A. et Morlaix, S. (2012). Les pratiques pédagogiques des enseignants universitaires : Quelle variété pour quelle efficacité ? Questions vives, 6(18), 93-110. https://doi.org/10.4000/questionsvives.1178

Fenelon, J. V. et LeBeau, D. (2005). Four directions for Indian Education: Curriculum models for Lakota/Dakota teaching and learning. Dans D. Champagne et I. AbuSaad (dir.), Indigenous and minority education: International perspectives on empowerment (p. 289-328). Negev Center for Regional Development.

Fiddler, C. (2015). The influence of Aboriginal literature on Aboriginal students' resilience at the University of Saskatchewan. Education Matters: The Journal of Teaching and Learning, 3(1), 1-16. https://journalhosting.ucalgary.ca/index.php/ em/article/view/62959/pdf_1

Fontaine, S. et Peters, M. (2012). L'abandon des étudiants à l'université : État de la question. Dans M. Romainville et C. Michaut (dir.), Réussite, échec et abandon dans l'enseignement supérieur (p. 33-52). De Boeck.

Fortin, M.-F. et Gagnon, J. (2016). Fondements et étapes du processus de recherche: Méthodes quantitatives et qualitatives (3e éd.). Chenelière éducation. https://doi. org/10.7202/1042088ar

Fraternité des Indiens du Canada. (1972). La maîtrise indienne de l'éducation indienne : déclaration de principe présentée au ministre des Affaires indiennes et du Nord 
canadien par la Fraternité des Indiens du Canada. https://www.afn.ca/uploads/ files/fn_education/icoie-fr.pdf

Frenay, M. (2006). 1.3.4 Deux visions du rôle de l'enseignant... Dans B. Raucent et C. Vander Borght (dir.), Etre enseignant: Magister? Metteur en scène? (p. 72-80). De Boeck Supérieur.

Fuentes, S. et Reyes, P. (2019). Pédagogie Holistique : la vraie bouée de sauvetage pour l'éducation publique ? Spécificités, 13(2), 229-237. https://doi.org/10.3917/ spec.013.0229

Gingras-Lacroix, G. (2019). Les pratiques enseignantes déclarées par les professeurs et les chargés de cours en contexte de classe incluant des étudiants autochtones universitaires [Mémoire de maitrise, Université du Québec en AbitibiTémiscamingue]. Depositum. https://depositum.uqat.ca/id/eprint/804

Giroux, H. A. (2010). Bare pedagogy and the scourge of neoliberalism: Rethinking higher education as a democratic public sphere. The Educational Forum, 74(3), 184-196. https://doi.org/10.1080/00131725.2010.483897

Goodman, R. D., Williams, J. M., Chung, R. C.-Y., Talleyrand, R. M., Douglass, A. M., McMahon, H. G. et Bemak, F. (2015). Decolonizing traditional pedagogies and practices in counseling and psychology education: A move towards social justice and action. Dans R. D. Goodman et P. C. Gorski (dir.), Decolonizing "multicultural" counseling through social justice (p. 147-164). Springer. https:// doi.org/10.1007/978-1-4939-1283-4

Irwin, B. et Hepplestone, S. (2012). Examining increased flexibility in assessment formats. Assessment \& Evaluation in Higher Education, 37(7), 773-785. https:// doi.org/10.1080/02602938.2011.573842

Johanne, J. (2020, novembre). L'action des universités québécoises pour, par et avec les Premiers Peuples - Portrait 2019. https://www.bci-qc.ca/wp-content/ uploads/2021/02/Portrait-2019 Premiers-Peuples_FR.pdf

Joncas, J.-A. (2013). Apport à la compréhension de l'expérience scolaire de persévérants universitaires des Premières Nations au Québec: le cas d'étudiants de l’Université du Québec à Chicoutimi [Mémoire de maitrise, Université du Québec à Chicoutimi]. Constellation. https://constellation.uqac.ca/2739/ 
Joncas, J.-A. (2018). La justice aux études supérieures : l'incidence du contexte d'études sur la réalisation de la carrière scolaire de femmes autochtones universitaires [Thèse de doctorat, Université Laval]. Corpus. http://hdl.handle. net/20.500.11794/31148

Kermoal, N. (2018). Le rôle des universités canadiennes dans la décolonisation des savoirs : le cas de l'Alberta. ACME : An International Journal for Critical Geographies, 17(3), 651-670. https://acme-journal.org/index.php/acme/article/ view/1260

Kold, D. A. (1986). Experiential learning: Experience as the source of learning and development. Prentice Hall.

Langevin, K. (2006). L'adaptation des étudiants autochtones au collégial [Mémoire de maitrise, Université du Québec à Chicoutimi]. Constellation. https://constellation. uqac.ca/444/1/24967644.pdf

Lee, D. H. L., Hong, H. et Niemi, H. (2014). A contextualized account of holistic education in Finland and Singapore: Implications on Singapore educational context. The Asia-Pacific Education Researcher, 23(4), 871-884. https://doi. org/10.1007/s40299-014-0189-y

Lefevre-Radelli, L. (2019). L'expérience des étudiants autochtones à l'université : racisme systémique, stratégies d'adaptation et espoir de changement social [Thèse de doctorat, Université de Nantes]. Nantes. https://www.theses. $\underline{\text { fr/2019NANT2039 }}$

Lévesque, C., Polèse, G., de Juriew, D., Labrana, R., Turcotte, A.-M. et Chiasson, S. (2015). Une synthèse des connaissances sur la réussite et la persévérance scolaires des élèves autochtones au Québec et dans les autres provinces canadiennes (Cahier no 2015-01) [Rapport de recherche]. Centre Urbanisation Culture Société; Réseau DIALOG. https://reseaudialog.ca/wp-content/ uploads/2019/12/CahierDIALOG2015-01-Réussitepersérancescolaires-Levesque2015-taille-réduite.pdf

Lincoln, Y. S. et Guba, E. G. (1985). Naturalistic inquiry. SAGE. 
Lison, C. (2011). Programmes innovants en formation des enseignants du secondaire : perceptions, conceptions et pratiques [Thèse de doctorat, Université de Sherbrooke]. Savoirs UdeS. http://savoirs.usherbrooke.ca/handle/11143/5822

Lison, C. et Jutras, F. (2014). Innover à l'université : penser les situations d'enseignement pour soutenir l'apprentissage [Éditorial]. Revue Internationale de Pédagogie de l'Enseignement Supérieur, 30(1), 1-8. https://doi.org/10.4000/ripes.769

Lloyd, N. J., Lewthwaite, B. E., Osborne, B. et Boon, H. J. (2015). Effective teaching practices for Aboriginal and Torres Strait Islander students: A review of the literature. Australian Journal of Teacher Education, 40(11), 1-22. https://doi. org/10.14221/ajte.2015v40n11.1

Loiselle, M. et Legault, L. (2010, février). Une analyse des déterminants de persévérance et de réussite des étudiants autochtones à l'Université du Québec en AbitibiTémiscamingue [Rapport de recherche]. Université du Québec en AbitibiTémiscamingue. https://esp.umontreal.ca/fileadmin/esp/documents/Cheminement/ soutien-reussite/Autochtones-etudes\%20supérieures.pdf

Malatest, R. A. (2002, mai). Pratiques exemplaires permettant d'accroître les taux de scolarisation postsecondaire des autochtones [Rapport]. http://www.cmec.ca/ publications/lists/publications/attachments/49/malatest.fr.pdf

Marcel, J.-F. (2009). De la prise en compte des pratiques enseignantes de travail partagé. Nouveaux cahiers de la recherche en éducation, 12(1), 47-64. https://doi. org/10.7202/1017487ar

Mareschal, J. et Denault, A.-A. (2020). Persévérance et réussite scolaires des étudiants autochtones au collégial : récits et pratiques liés à la sécurisation culturelle issus de cégeps de Québec et de Trois-Rivières. Cégep Garneau. https://numerique.banq. qc.ca/patrimoine/details/52327/4209349? docref $=\mathrm{iUhg} 3 \mathrm{HqN}$ HHfl5Nkepf2BA

Mayes, C., Cutri, R. M., Goslin, N. et Montero, F. (2016). Understanding the whole student: Holistic multicultural education (2e éd.). Rowman \& Littlefield.

Michaut, C. (2012). Réussite, échec et abandon des études dans l'enseignement supérieur français : quarante ans de recherche. Dans M. Romainville et C. Michaut (dir.), Réussite, échec et abandon dans l'enseignement supérieur (p. 53-68). De Boeck. 
Miller, J. P., Karsten, S., Denton, D., Orr, D. et Colalillo Kates, I. (dir.). (2005). Holistic learning and spirituality in education: Breaking new ground. SUNY Press.

Milne, T., Creedy, D. K. et West, R. (2016). Integrated systematic review on educational strategies that promote academic success and resilience in undergraduate indigenous students. Nurse education today, 36, 387-394. https://doi. org/10.1016/j.nedt.2015.10.008

Moldoveanu, M. et Campeau, D. (2016). Les approches pédagogiques pour soutenir la réussite scolaire des élèves autochtones. Dans M. Potvin, M.-O. Magnan et J. Larochelle-Audet (dir.), La diversité ethnoculturelle, religieuse et linguistique en éducation (p. 197-205). Fides Éducation.

Morandi, F. et La Borderie, R. (2001). Modèles et méthodologies en pédagogie (2e éd.). Nathan. https://doi.org/10.3917/nath.moran.2001.01

Mostrom, A. M. et Blumberg, P. (2012). Does learning-centered teaching promote grade improvement? Innovative Higher Education, 37(5), 397-405. https://doi. org/10.1007/s10755-012-9216-1

Mucchielli, A. (2009). Méthode de l'analyse phénoménologique. Dans A. Mucchielli (dir.), Dictionnaire des méthodes qualitatives en sciences humaines (3e éd., p. 290-291). Armand Colin.

Munroe, E. A., Lunney Borden, L., Murray Orr, A., Toney, D. et Meader, J. (2013). Decolonizing Aboriginal education in the 21 st century. McGill Journal of Education, 48(2), 317-338. https://doi.org/10.7202/1020974ar

Nardozi, A., Restoule, J.-P., Broad, K., Steele, N. et James, U. (2014). Deepening knowledge to inspire action: Including Aboriginal perspectives in teaching practice. In education, 19(3), 108-122. https://doi.org/10.37119/ojs2014. v19i3.140

Nayyar, D. (2008). Globalization: What does it mean for higher education? Dans L. E. Weber et J. J. Duderstadt (dir.), The globalization of higher education (p. 3-14). Economica.

Paillé, P. et Mucchielli, A. (2003). L'analyse qualitative en sciences humaines et sociales. Armand Colin. 
Papillon, M. et Cosentino, G. (2004, avril). Lessons from abroad: Towards a new social model for Canada's Aboriginal peoples (Rapport no F40) [Rapport de recherche]. Canadian Policy Research Networks.

Pires, A. P. (1997). Échantillonnage et recherche qualitative : essai théorique et méthodologique. Dans J. Poupart, J.-P. Deslauriers, L. H. Groulx, A. Laperrière, R. Mayers et A. P. Pires (dir.), La recherche qualitative : enjeux épistémologiques et méthodologiques (p. 113-172). Gaëtan Morin.

Price, R. et Burtch, B. (2010). Degree completion for Aboriginal people in British Columbia: A case study. Canadian Journal of University Continuing Education, 36(1), 1-17. https://doi.org/10.21225/d5wc7m

QSR International Pty Ltd. (2014). Nvivo (Version 10) [Logiciel]. https://www. qsrinternational.com/nvivo-qualitative-data-analysis-software/home

Ricci, C. et Pritscher, C. P. (2015). Holistic pedagogy: The self and quality willed learning. Springer.

Robert-Careau, F. (2019). La socialisation scolaire des jeunes autochtones au Québec : l'exemple du cégep de Joliette [Mémoire de maitrise, Université Laval]. Corpus. http://hdl.handle.net/20.500.11794/33745

Rodon, T. et Tremblay, M.-L. (2007, octobre). Les étudiants autochtones à l'Université Laval : analyse des besoins et évaluation des stratégies d'accueil et de soutien [Rapport]. Université Laval, Faculté des sciences sociales. http://pages.ccapcable. com/thierry_rodon/TR/Recherche_Research_files/rapportfinal.pdf

Romainville, M. et Michaut, C. (dir.). (2012). Réussite, échec et abandon dans l'enseignement supérieur. De Boeck.

Sanford, K., Williams, L., Hopper, T. et McGregor, C. (2012). Indigenous principles decolonizing teacher education: What we have learned. In education, 18(2), 19-34. https://doi.org/10.37119/ojs2012.v18i2.61

Savard, J. (2010). Les femmes autochtones et l'éducation postsecondaire [Mémoire de maitrise, Université du Québec à Montréal]. Archipel. https://archipel.uqam. ca/9489/1/M11766.pdf 
Savoie-Zajc, L. (2006, 16 mai). Comment peut-on construire un échantillonnage scientifiquement valide? Dans F. Guillemette et C. Baribeau (dir.), Actes $d u$ colloque de l'Association pour la recherche qualitative organisé dans le cadre du congrès de l'ACFAS (p. 99-111). Association pour la recherche qualitative. http:// www.recherche-qualitative.qc.ca/documents/files/revue/hors_serie/hors_serie_v5/ RQ-HS-5-Numero-complet.pdf

Savoie-Zajc, L. (2009). Technique de validation par triangulation. Dans A. Mucchielli (dir.), Dictionnaire des méthodes qualitatives en sciences humaines (3e éd., p. 285-286). Armand Colin.

Savoie-Zajc, L. (2010). L'entrevue semi-dirigée. Dans B. Gauthier (dir.), Recherche sociale : de la problématique à la collecte des données (5e éd., p. 337-360). Presses de l'Université du Québec.

Shield, R. W. (2004). The retention of indigenous students in higher education: Historical issues, federal policy, and indigenous resilience. Journal of College Student Retention: Research, Theory \& Practice, 6(1), 111-127. https://doi.org/10.2190/ hwkh-81c1-wp90-45k0

Sioui, B. (2012). Le regard critique des jeunes Autochtones d'Abitibi-Est sur les conditions favorables à leur persévérance scolaire et à leur réussite éducative. Inditerra, Revue internationale sur l'Autochtonie, 4, 1-13.

Sioui, G. (2008). Histoires de Kanatha, vues et contées : essais et discours, 1991-2008. Presses de l'Université d'Ottawa.

St-Onge, M. (2016). L'évolution des finalités de l'université québécoise dans un contexte de mondialisation : analyse critique des politiques publiques de 1998 à 2009 [Thèse de doctorat, Université de Montréal]. Papyrus. https://papyrus.bib. umontreal.ca/xmlui/bitstream/handle/1866/13512/St-Onge_Marianne_2015 these.pdf

Statistique Canada. (2012, février). Classification nationale des professions (no 12-583X). Ministre de l'Industrie. https://www150.statcan.gc.ca/n1/pub/12-583-X/12583-x2011001-fra.pdf

Statistique Canada. (2016). Répartition de la population âgée de 25 à 64 ans (total et ayant une identité autochtone), selon le plus haut certificat, diplôme ou grade 
et selon le groupe d'âge (Tableau 37-10-0099-01). Gouvernement du Canada. https://doi.org/https://doi.org/10.25318/3710009901-fra

Styres, S., Haig-Brown, C. et Blimkie, M. (2013). Towards a pedagogy of land: The urban context. Canadian Journal of Education, 36(2), 34-67. https://journals.sfu. ca/cje/index.php/cje-rce/article/view/1293

Thibeault, J. (2015, février). La démographie des Premières Nations. Le Bulletin, 2-3.

Timmons, V., Doyle-Bedwell, P. et Lewey, L., Marshall, L., Power, B., Sable, T. et Wien, F. (2009, juin). Retention of Aboriginal students in post-secondary institutions in Atlantic Canada: An analysis of the supports available to Aboriginal students Research project 2007-2009. Association of Atlantic Universities. https://www. atlanticuniversities.ca/sites/default/files/documents/AAUNews2009Archive/ Retention $\% 20$ of $\% 20$ Aboriginal $\% 20$ Students $\% 20$ in $\% 20$ Post $\% 20$ Secondary $\% 20$ Institutions.pdf

Töytäri, A., Tynjälä, P., Piirainen, A. et Ilves, V. (2017). Higher education teachers' descriptions of their own learning: a quantitative perspective. Higher Education Research \& Development, 36(6), 1295-1304. https://doi.org/10.1080/07294360.2 $\underline{017.1303455}$

Troop, M. A. C., Wallar, L. E. et Aspenlieder, E. (2015). Developing graduate students' self-efficacy with learner-centred lecturing. Canadian Journal of Higher Education, 45(3), 15-33. https://doi.org/10.47678/cjhe.v45i3.187548

Van der Maren, J.-M. (2004). Méthodes de recherche pour l'éducation (2e éd.). De Boeck.

Walder, A. M. (2015). Obstacles to innovation: The fear of jeopardising a professorial career? British Journal of Education, 3(6), 1-16. https://www.eajournals. org/wp-content/uploads/Obstacles-to-innovation-The-fear-of-jeopardising-aprofessorial-career.pdf

Walton, P., Hamilton, K., Clark, N., Pidgeon, M. et Arnouse, M. (2020). Indigenous university student persistence: Supports, obstacles, and recommendations. Canadian Journal of Education, 43(2), 430-464. https://journals.sfu.ca/cje/index. php/cje-rce/article/view/3975 
Wanner, T. et Palmer, E. (2015). Personalising learning: Exploring student and teacher perceptions about flexible learning and assessment in a flipped university course. Computers \& Education, 88, 354-369. https://doi.org/10.1016/j. compedu.2015.07.008

Watkins, E. (2008). Class degrees: Smart work, management choices and the transformation of higher education. Fordham University Press. https://doi. org/10.2307/j.ctt13wzz28

White, F. H. (2001). Participation and learning styles of the haida: a study of a haida language class (UMI no 3026263) [Dissertation, University of California]. ProQuest Dissertation \& Theses. 